\title{
Dinitrosyl Iron Complexes with Thiol-Containing Ligands Can Suppress Viral Infections as Donors of the Nitrosonium Cation (Hypothesis)
}

\author{
A. F. Vanin ${ }^{a, b}$, * \\ ${ }^{a}$ Semenov Institute of Chemical Physics, Moscow, 119334 Russia \\ ${ }^{b}$ Institute of Regenerative Medicine, Sechenov First Moscow State Medical University, Moscow, 119991 Russia \\ *e-mail:vanin@polymer.chph.ras.ru \\ Received April 6, 2020; revised May 8, 2020; accepted May 12, 2020
}

\begin{abstract}
The appropriateness of verification of the possible antiviral effect of dinitrosyl iron complexes with thiol-containing ligands as donors of nitrosonium cations $\left(\mathrm{NO}^{+}\right)$is argued. There is reason to hope that treatment of the human respiratory tract and lungs with sprayed solutions of dinitrosyl iron complexes with glutathione or $\mathrm{N}$-acetylcysteine (NAC) as $\mathrm{NO}^{+}$donors during COVID-19 infection can initiate S-nitrosylation of cellular proteases and thereby suppress viral infection.
\end{abstract}

Keywords: dinitrosyl iron complexes, nitrosonium, S-nitrosylation, viral infections

DOI: $10.1134 / \mathrm{S} 0006350920040260$

DINITROSYL IRON COMPLEXES WITH THIOL-CONTAINING LIGANDS: PHYSICOCHEMICAL AND BIOLOGICAL CHARACTERISTICS

It has been established that all representatives of the living world, that is, animals and humans, plants and microorganisms, continuously produce the simple compound nitric monoxide (or simply nitric oxide, NO) using enzymes, which functions in living organisms as one of the universal regulators of various metabolic and physiological processes [1-3]. In addition, as a rule, at elevated concentrations (up to $100 \mu \mathrm{mol} / \mathrm{kg}$ animal weight), nitric oxide acts as one of the main effectors of the cellular immunity system.

There is reason to believe that the functioning of $\mathrm{NO}$ as an autocrine and especially paracrine effector in animals and humans is provided by its incorporation into dinitrosyl iron complexes (DNICs) with thiol-containing $\left(\mathrm{RS}^{-}\right)$ligands [4-6]. These complexes, which exist in mononuclear and binuclear forms (M-DNICs and B-DNICs, respectively) and are described by the formulas $\left[\left(\mathrm{RS}^{-}\right)_{2} \mathrm{Fe}(\mathrm{NO})_{2}\right]$ and $\left[\left(\mathrm{RS}^{-}\right)_{2} \mathrm{Fe}_{2}(\mathrm{NO})_{4}\right]$, respectively, arise in cells and tissues of animals that produce $\mathrm{NO}$ and completely mimic the biological activity of this agent [5-8]. Concerning the latter, it seems appropriate to talk not only about neutral NO molecules, but about the system of

Abbreviations: DNICs, dinitrosyl iron complexes; M-DNICs, mononuclear dinitrosyl iron complexes; B-DNICs, binuclear dinitrosyl iron complexes. their derivatives that are responsible for various metabolic and physiological processes, i.e., about the biological system of nitric oxide. There is reason to believe that $\mathrm{M}$ - and B-DNICs with thiol-containing ligands act in living organisms as an essential component of this system. This assumption is based on three types of experimental data concerning these complexes. The first is the large amount of data, which was mentioned above, that indicate a diverse biological effect of the chemically synthesized (exogenous) Mand B-DNICs that mimic the biological activity of the endogenous NO system [5-8]. The second type is evidence that nitric oxide in animal tissues is almost completely included in B-DNICs, which provides its stabilization and, obviously, transfer of NO to targets of its biological effect $[9,10]$. The third type of data demonstrates the ability of both forms of DNIC to initiate the conversion of neutral NO molecules included in these complexes into nitrosonium $\left(\mathrm{NO}^{+}\right)$cations [11-13]. As a result, dinitrosyl iron complexes with thiol-containing ligands, as donors of these cations, are capable of causing S-nitrosylation of high- and low-molecular-weight thiol-containing compounds, i.e., imitate one of the most important manifestations of biological activity of the endogenous NO system: initiation of the formation of S-nitrosothiols in living organisms.

It should be noted that in contrast to the mononuclear paramagnetic form of DNICs with thiol-containing ligands, as characterized by an electron paramagnetic resonance signal with $g_{\text {sr }}=2.03$ (the so- 
called 2.03 signal), B-DNICs with thiol-containing ligands are diamagnetic, i.e., they are EPR-inactive [14]. Nevertheless, their presence in animal tissues can be detected by treating the tissues both in vivo and in vitro with derivatives of dithiocarbamate (diethyldithiocarbamate or N-methyl-D-glucamine dithiocarbamate), which is capable of accepting mononitrosyliron $([\mathrm{Fe}(\mathrm{NO})])$ fragments from iron dinitrosyl $\left(\left[\mathrm{Fe}(\mathrm{NO})_{2}\right]\right)$ groups of DNICs with the formation of EPR-recorded mononitrosyl iron complexes with dithiocarbamate derivatives $[9,10]$.

DNICs with thiol-containing ligands and another form of NO stabilization in living organisms, S-nitrosothiols (general formula is RS-NO), are connected, i.e., they define each other's existence. This is mani- fested in the fact that both DNICs can arise from RS$\mathrm{NO}$, and the latter can occur in living organisms as a result of the release of nitrosonium cations from dinitrosyl complexes, whose interaction with thiols leads to the formation of RS-NO [5, 6].

In accordance with the ideas developed by our group on the mechanisms of DNIC formation with the participation of molecular NO or RS-NO, in both cases these mechanisms are determined by the disproportionation reaction of $\mathrm{NO}$ or RS-NO, i.e., the oneelectron mutual oxidation-reduction reaction of these agents, which occurs after the binding of NO or RSNO with ferrous ions (two molecules per iron ion), see diagrams 1 and 2:

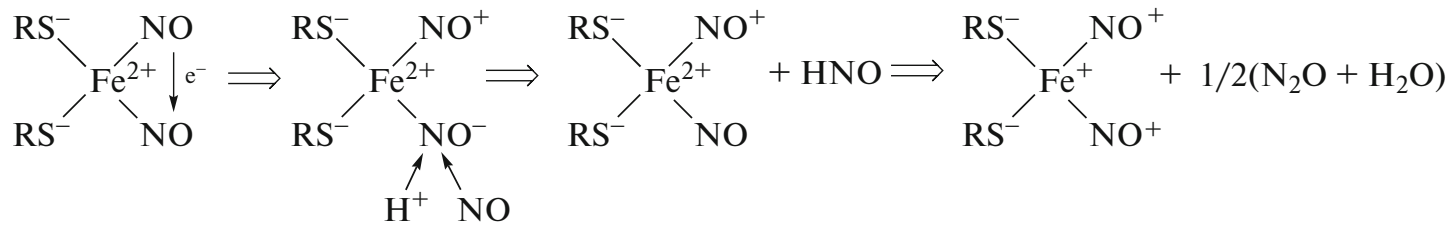

Diagram 1. The proposed scheme for the formation of M-DNICs with thiol-containing ligands in the reaction of ferrous iron, thiols, and gaseous NO [4-6, 15-17].

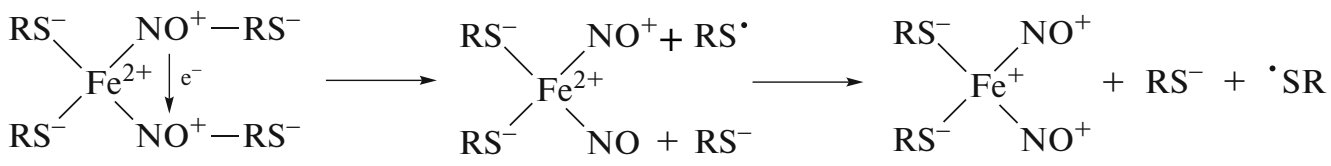

Diagram 2. The alleged mechanism of the formation of M-DNICs with thiol-containing ligands in the reaction of Fe ${ }^{2+}$, thiols, and RS-NO [15-18].

The mechanisms of the formation of M-DNICs with thiol-containing ligands presented in Diagrams 1 and 2 were considered in detail in our previous publications [15-18]. It should be noted here that in both cases complexes with the same electronic and spatial structures are formed, as characterized by the electronic configuration of iron $d^{7}$ with the corresponding resonant structure $\left[\left(\mathrm{RS}^{-}\right)_{2} \mathrm{Fe}^{+}\left(\mathrm{NO}^{+}\right)_{2}\right]^{+}$.

For the binuclear form of DNIC (B-DNICs) with thiol-containing ligands, it has been shown that it is formed by the condensation of two mononuclear forms of these complexes [14], regardless of the methods of synthesis of the latter with a decrease in the content of thiol-containing ligands in the solution in accordance with the reversible interconversion scheme M- and B-DNICs (Diagram 3) and is characterized by a resonant structure that is similar to the structure of M-DNIC, that is $\left[\left(\mathrm{RS}^{-}\right)_{2} \mathrm{Fe}_{2}^{+}\left(\mathrm{NO}^{+}\right)_{4}\right]^{+4}$ :

$$
\text { M-DNIC } \stackrel{-\mathrm{RS}^{-}}{\rightleftarrows} \text { B-DNIC }
$$

Diagram 3. Reversible interconversion of Mand B-DNICs with thiol-containing ligands [14].

Based on the above resonance structure of MDNICs, the chemical equilibrium between these complexes and their constituent components can be represented in accordance with Diagram 4 as

$$
\left[\left(\mathrm{RS}^{-}\right)_{2} \mathrm{Fe}^{+}\left(\mathrm{NO}^{+}\right)_{2}\right] \Longleftrightarrow \mathrm{Fe}^{2+}+\mathrm{NO}+\frac{\mathrm{RS}^{-}+\mathrm{NO}^{+}}{\mathrm{RS}-\mathrm{NO}}+\mathrm{RS}^{-}
$$

Diagram 4. Chemical equilibrium between M-DNICs with thiol-containing ligands and their constituent components [15-18].

Exactly the same components of this set of constituent parts of M-DNIC components, that is, $\mathrm{Fe}^{2+}$,
NO, RS-NO, and thiols $\left(\mathrm{RS}^{-}\right)$, can be released from these complexes depending on the mechanism of their 
decomposition. Thus, during decomposition caused by the removal of ferrous iron from M-DNICs under the action of the corresponding iron chelators, molecules of NO and nitrosonium cations should obviously be transferred into the solution in a free state, whose hydrolysis is prevented by their inclusion in RS-NO. This assumption is consistent with the results of the studies of our group regarding the interaction of DNICs with dithiocarbamate derivatives that intercept iron-mononitrosyl fragments from these complexes [19]. On the other hand, during decomposition caused by the removal of NO molecules from the complexes [20] as a result of their incorporation into more stable nitrosyl complexes of heme iron or as a result of the binding of NO molecules to superoxide anions [21], $\mathrm{Fe}^{2+}$ ions and nitrosonium cations (more precisely, RS-NO) should obviously go into solution in a free state.

\section{How M- and B-DNICs with Thiol-Containing Ligands Can Suppress Virus Replication in Tissues and Cells of a Host}

In the 1980-1990s, immediately after establishing the role of NO as one of the main effectors of the cellular immunity system, an intensive study of the effect of this agent and its donors on the development of various infectious pathologies, including those of viral origin, began. Hundreds of articles and many reviews have been published, in which it was shown that most pathologies caused by viral infection in animals and humans are characterized by a sharp increase in the level of nitric oxide in the host cells and tissues (mainly as a result of activation of synthesis of the inducible NO synthase: iNOS or NOS2), reactive oxygen species (ROS), as well as a sharp increase in the concentration of various cytokines [22-32]. It has been established that an increase in the level of NO suppresses the replication of viral RNA and DNA in host cells, leading to the deactivation of the most important viral proteins that are necessary for the reproduction of viruses, such as viral proteases, reverse transcriptases, and transcription factors; this deactivation is initiated by S-nitrosylation of functionally important thiol groups in these proteins. A similar result was obtained in experiments using exogenous NO donors, that is, S-nitroso-N-acetylpenicillamine (SNAP), NONOates, sodium nitroprusside, and even nitroglycerin.

It is possible that during some viral infections, $\mathrm{S}$-nitrosylation of nonviral proteins, the host proteins, for example, proteinases, can suppress the initial stage of infection. The latter can occur during viral infections in which contact and subsequent fusion of viruses and host cells can occur with the participation of the corresponding host proteins.

In 2006 a study was published [33] in which it was shown that M-DNICs with cysteine alone and especially that attached to the cysteine residue of the tetra- peptide Leu-Ser-Tre-Cis selectively bind to the protease 2A of Coxsackie B virus and completely inhibit the activity of this enzyme. This inhibition, observed in experiments on isolated protease $\mathrm{A}$, as well as on protease A included in cell cultures, was due to S-nitrosylation of one of the thiol groups of this enzyme and was completely reversible. When S-nitrosylated protease $2 \mathrm{~A}$ was treated with dithiothreitol intercepting nitrosonium cations that were part of protein S-nitrosothiols to its thiol groups, the activity of protease A was completely restored. Similarly, M-DNICs with cysteine alone initiated S-nitrosylation of intracellular caspase 3.

It was noted that $\mathrm{S}$-nitrosylation of protease $2 \mathrm{~A}$ in murine myocardial tissues significantly attenuated viral infection [34].

As the authors of [33] suggested, the ability of MDNICs with cysteine to cause S-nitrosylation of protease $2 \mathrm{~A}$ was due to the existence of both nitrosyl ligands in the iron-dinitrosyl groups of these complexes in the form of nitrosonium cations, which initiated the formation of S-nitrosothiols as a part of protease $2 \mathrm{~A}$ and caspase 3 . This assumption is only partially true. In accordance with the results of our studies of DNICs with thiol-containing ligands, these complexes can act not only as donors of nitrosonium cations that provide S-nitrosylation of thiols, but also as donors of neutral NO molecules; as a rule, these molecules are released from DNICs in an amount approximately equal to the amount of released nitrosonium cations [5-8, 15-18]. This phenomenon is provided by the transfer of an electron from an iron atom to one of the nitrosyl ligands in the resonance structure of M-DNICs, $\left[\left(\mathrm{RS}^{-}\right)_{2} \mathrm{Fe}^{+}\left(\mathrm{NO}^{+}\right)_{2}\right]^{+}$, and its transition to the structure $\left[\left(\mathrm{RS}^{-}\right)_{2} \mathrm{Fe}^{2+}\left(\mathrm{NO}^{+}\right)(\mathrm{NO})\right]^{+}$.

How can S-nitrosylation of viral proteins in host cells during a viral infection be carried out when lowmolecular-weight DNICs with thiol-containing ligands are included in these cells? Some of these ligands (M-DNICs with cysteine) were used by the authors of [33]. The results of many studies of the behavior of low-molecular-weight DNICs with thiolcontaining ligands, as they were introduced into protein-containing solutions, in cell cultures or in the animal organism, show that in these systems a rapid transfer of iron-dinitrosyl groups from these complexes to thiol groups of proteins occurs with the formation of protein DNICs. These complexes can incorporate not only two thiol-containing (protein) ligands, two cysteine residues, but also include only one of these residues as a thiol-containing ligand, whereas, a second, a nitrogen-containing ligand, histidine residues, for example, can be included in protein DNICs $[35,36]$. Nevertheless, a single cysteine residue may be sufficient for S-nitrosylation of this protein. How will this transformation be carried out, that is, the transition, for example, of a viral protease, 
a protein containing DNA associated with it, into a protein with S-nitrosylated cysteine residues?

As mentioned in the previous section, S-nitrosylation of thiols caused by the release of nitrosonium cations from DNICs with thiol-containing ligands can be due to the decomposition of these complexes either as a result of the removal of iron from these complexes under the action of appropriate iron chelators, or as a result of the binding to superoxide anion of half nitrosyl ligands represented in DNICs in the form of neutral molecules. This type of breakdown of protein DNICs can occur naturally in cells infected with viruses without the addition of exogenous iron chelators or donors of superoxide radicals. First, under conditions of intensive replication of viruses in host cells, it becomes necessary to deliver iron to these cells, which can be achieved by the production of endogenous iron chelators that are capable of destroying protein DNICs. Secondly, superoxide anions in host cells can occur as a reaction to the inflammatory processes caused by a viral infection. In addition, superoxide radicals can occur as a result of oxygen oxidation of ferrous ions released from DNICs by positive feedback mechanism (by the "explosive" mechanism).

There is reason to believe that the use of lowmolecular-weight DNICs with thiol-containing ligands in the treatment of coronavirus can provide the greatest positive effect when inhaled as sprayed (passed through a nebulizer) aqueous DNIC solutions. The greatest effect, naturally, is expected from complexes that most effectively penetrate into epithelial cells of tissues of the respiratory tract and lungs. DNICs with N-acetyl-L-cysteine can be recommended as such complexes, which relatively easily penetrate the cells [13].

In conclusion, it should be noted that the use of gaseous NO, as well as agents that release NO, that is, $\mathrm{S}$-nitrosothiols or NONOates, can lead to the formation of, first, DNICs with low-molecular-weight thiol-containing ligands (primarily with glutathione) and then with protein thiols in epithelial cells and lung cells affected by coronavirus (in accordance with Diagrams 1 and 2 with the participation of loosely bonded intracellular iron and endogenous thiols). The mechanism of S-nitrosylation of viral proteins can then be carried out according to the mechanism described above.

Thus, DNICs with thiol-containing ligands as donors of nitrosonium cations that are capable of initiating S-nitrosylation of viral and host proteins can suppress viral infection both at its initial stage and at the stage of intracellular replication of viruses. For blockade of viral infection by neutral NO molecules released from DNICs under the action of, for example, iron chelators on them, in contrast to $\mathrm{NO}^{+}$cations, which weakly affect the host cells [22-32], NO molecules that turn into very toxic peroxynitrite in the reactions with superoxide can have a toxic effect on host cells more efficiently than on pathogenic viruses [37-45].

\section{ACKNOWLEDGMENTS}

The author is grateful to Dr. S. Yakovenko for providing technical assistance without which it would be impossible to write this article in the conditions of a COVID-19 quarantine.

\section{FUNDING}

This work was carried out within the framework of the State Assignment of the Ministry of Education and Science for research institutions (00008202014-0001b no. AAAAA17-117040610310-b, 0082-2014-0008 and AAAA-A171170403100008-5) and sponsored by the "5-100" Russian academic project and was also financed under grant of the Russian Foundation for Basic Research no. 18-04-00059a.

\section{COMPLIANCE WITH ETHICAL STANDARDS}

In carrying out this work, all ethical standards were observed.

Conflict of Interest. The authors declare that they have no conflicts of interest.

This article does not contain any studies involving humans and animals as objects.

\section{REFERENCES}

1. L. J. Ignarro, Nitric Oxide: Biology and Pharmacology (Academic, San Diego, 2000).

2. P. Domingos, A. M. Prado, A.Wong, et al., Mol. Plant. 8, 506 (2015).

3. A. M. Stem, J. Zhu, Appl. Microbiol. 87, 187 (2014).

4. A. F. Vanin, Nitric Oxide Biol. Chem. 54, 15 (2016).

5. A. F. Vanin, Dinitrosyl Iron Complexes as a "Working Form"of Nitric Oxide in Living Organisms (Cambridge Scholars Publ., Cambridge, UK, 2019).

6. A. F. Vanin, Dinitrosyl Iron Complexes with Thiol-containing Ligands: Physicochemistrey, Biology, Medicine (Inst. Comput. Res., Izhevsk, 2015) [in Russian].

7. A. F. Vanin, The Open Conf. Proc. J. 4, 23 (2013).

8. A. F. Vanin, Cell Biochem. Biophys. 76, 3 (2018).

9. A. F. Vanin, V. D. Mikoyan, L. N. Kubrina, et al., Biophysics (Moscow) 60 (4), 603 (2015).

10. V. D. Mikoyan, E. N. Burgova, R. R. Borodulin, et al., Nitric Oxide Biol. Chem. 62, 1 (2017).

11. A. F. Vanin, Austin J. Analyt. Pharmac. Chem. 5 (4), 1109 (2018).

12. A. F. Vanin, Cell Biochem. Biophys. 77, 279 (2019).

13. A. F. Vanin, Biophysics (Moscow) 65 (3), 421 (2020).

14. A. F. Vanin, A. P. Poltorakov, V. D. Mikoyan, et al., Nitric Oxide Biol. Chem. 23, 136 (2010).

15. A. F. Vanin, I. V. Malenkova, and V. A. Serezhenkov, Nitric Oxide Biol. Chem. 1, 191 (1997).

16. A. F. Vanin, Nitric Oxide Biol. Chem. 21, 1 (2009). 
17. A. F. Vanin and D. Sh. Burbaev, Biophys. J. 14, 818 (2011).

18. A. F. Vanin, A. A. Papina, V. A. Serezhenkov, et al., Nitric Oxide Biol. Chem. 10, 60 (2004).

19. R. R. Borodulin, L. N. Kubrina, V. D. Mikoyan, et al., Nitric Oxide Biol. Chem. 29, 4 (2013).

20. N. A. Sanina, L. A. Syrtsova, N. I. Shkondina, et al., Nitric Oxide Biol. Chem. 16, 181 (2007).

21. K. B. Shumaev, A. A. Gubkin, V. A. Serezhenkov, et al., Nitric Oxide Biol. Chem. 18, 37 (2008).

22. J. B. Mannick, K. Asano, and K. Izumi, Cell 79, 1137 (1994).

23. D. G. Karupiah and N. Harris, J. Exp. Med. 181, 2172 (1995).

24. J. MacMicking, A-W. Xie, and C. Nathan, Annu. Rev. Immunol. 15, 323 (1995).

25. C. S. Reiss and T. Kamatsu, J. Virol. 72, 4547 (1998).

26. S. P. Sanders, Proc. Soc. Exp. Biol. Med. 220, 123 (1999).

27. G. F. Rimmelzwaan, M. M. J. W. Baars, P. deLuster, et al., J. Virol. 73, 8880 (1999).

28. C. Tafalla, A. Figneras, and B. Novos, Vet. Immunol. Immunopathol. 72, 249 (1999).

29. T. Akaike and H. Maeda, Immunology 101, 300 (2000).

30. M. S. Finkel, Circulation 102, 2162 (2000).

31. E. Keyaerts, L. Vijgen, L. Chen, et al., Int. J. Infect. Dis. 8, 223 (2004).

32. M. S. Abdul-Cader, A. Amarasingh, M. R. Abdul-Careem, et al., Arch. Virol. 161, 2075 (2016).
33. C. Badorff, B. Fichtlscherer, A. Muelsch, et al., Nitric Oxide Biol. Chem. 6, 305 (2002).

34. C. Badorff, B. Fichtlscherer, R. E. Rhoads, et al., Circulation 102, 162 (2000).

35. A. F. Vanin, V. A. Serezhenkov, V. D. Mikoyan, et al., Nitric Oxide Biol. Chem. 2, 224 (1998).

36. D. R. Truzzi, S. V. Alves, L. E. S. Netto, and O. Augusto, Antioxidants 9, 276 (2020).

37. G. Karupiah, J-H. Chen, S. Mahalingam, et al., J. Exp. Med. 188, 1541 (1999).

38. P. Koka, K. He, J. A. Zack, et al., J. Exp. Med. 182, 941 (1995).

39. T. Akaike, Y. Noguchi, S. Ijiri, et al., Proc. Natl. Acad. Sci. U. S. A. 93, 244 (1996).

40. S. Mihm, A. Fayyzi, and G. Ramadori, Hepatology 26, 451 (1997).

41. P. L. Majano, M. J. Borque, and R. Moren-Otero, J. Clin. Invest. 101, 1343 (1998).

42. C-F. Lin, H-Y. Lei, A-L. Shian, et al., J. Immunol. 169, 657 (2002).

43. X. Lim, M. Janas, and S. Dasguptas, J. Biol. Chem. 273, 39312 (2002).

44. K. Machida, K. T-H. Cheng, V. M-H. Sun, et al., J. Virol. 78, 8835 (2004).

45. M.-M. Wang, M. Lu, C-L. Zhang, et al., Mol. Med. Rep. 18, 1867 (2018). https://doi.org/10.3892/mmr.2018.9089

Translated by A. Ostyak 